\title{
Role of Continuous Lumbar Drainage in Cerebrospinal Fluid Leak: A Prospective Study
}

\author{
AHMED HOSAMELDIN, M.D.; ASHRAF A. OSMAN, M.D. and MOHAMED G. ABDEL TAWAB, M.D. \\ The Department of Neurosurgery, Faculty of Medicine, Fayoum University, Egypt
}

\begin{abstract}
Background: Cerebrospinal Fluid (CSF) leak is one of the most disturbing issues that face neurosurgeons.

Aim of Study: The aim of this study is to illustrate the efficacy of Continuous Lumbar Drainage (CLD) as a prophylactic and therapeutic method for CSF leak with assessment of clinical outcome and early post-operative sequelae.

Patients and Methods: This study was prospectively conducted on patients with traumatic or post-operative CSF leak and those susceptible for post-operative CSF leak as skull base and spinal intradural surgeries in the period from May 2015 to November 2015 at the Neurosurgery Departments of Cairo and Fayoum Universities. These patients were subjected to insertion of CLD.

Results: Excellent results in 15 patients who showed cessation of CSF leak within 4 days of CLD, good results in 4 patients who showed cessation of CSF leak within 5 days of CLD and fair results only with one patient required reexploration then showed cessation of CSF leak within 5 days of CLD.

Conclusion: The present analysis of the data reveals that CLD is used as a prophylactic measure and a line of treatment for CSF leak. It may be used pre, intra or post-operatively. This study recommends the use of CLD in cases with CSF leak or suspected post-operative CSF leak.
\end{abstract}

Key Words: Leak-Cerebrospinal fluid (CSF) - Lumbar drain.

\section{Introduction}

CEREBROSPINAL Fluid (CSF) is a major component of the brain and spinal cord and serves as a shock absorbing agent to protect the brain, cerebellum, and meninges. CSF, a transparent fluid, encloses the brain and meninx. It is produced daily by the ventricles mainly [1].

Generally, trauma is a major cause of CSF leak. It has been reported that CSF leak is caused by

Correspondence to: Dr. Ahmed Hosameldin, The Department of Neurosurgery, Faculty of Medicine, Fayoum University, Egypt direct trauma in $80 \%$ of cases, surgery in $16 \%$, and spontaneous in $4 \%$ of patients $[2,3]$.

CSF leak predisposes the patients to lifethreatening bacterial contamination that can cause threatening infections, especially meningitis [4].

CSF leak management requires a fundamental multidisciplinary approach. Until recently, the management of this condition was almost exclusively neurosurgical [5].

CLD is a commonly used procedure in neurosurgery for different objectives, such as in cases of subarachnoid hemorrhage to prevent vasospasm, to treat post-traumatic and postoperative CSF leak, and even to reduce inreased intracranial pressure [5]

Perioperative CSF diversion deccreases the incidence of leak post-operatively. Diversion can be used as a successful prophylactic measure and as a line of treatment for CSF leak (rhinorrhea) following trans-sphenoidal pituitary surgery [4]

CLD helps in preventing and/or treating CSF leaks and may abandon re-exploration surgery.

Complications of lumbar drains are not so common; but, lumbar drains can prolong the hospital stay. The essential complications are headache, nausea, vomiting and patient discomfort. Major potential morbidities include additional surgery, meningitis, cerebral edema, cerebral herniation and tension pneumoencephalus [4].

\section{Patients and Methods}

The study was prospectively conducted on patients diagnosed with CSF leak either traumatic or post-operative and those patients amenable to 
CSF leak after neurosurgical operations such as skull base and intradural spinal surgeries in the period from May 2015 to November 2015 in the Neurosurgery Departments of Cairo and Fayoum Universities.

This study included patients with post-operative CSF leak (cranial and spinal surgeries) and patients with post traumatic CSF leak.CLD was performed pre-operatively in patients highly amenable to postoperative CSF leak (skull base and spinal intradural surgeries).

Exclusion criteria were; patients with GCS $<8$, infection of surrounding skin, subcutaneous tissue, bone and epidural space, patients with bleeding disorders and patients on anticoagulants.

All patients were undergone thorough history taking, clinical examination and investigations with special attention to the following points: History of head trauma, fluid leak from nose or ear and presence of co-morbidities.

\section{Operative technique:}

In this study, all patients were subjected to continuous lumbar drain insertion. The patients were positioned in either the lateral decubitus or sitting with flexing the trunk anteriorly in the operation room under monitoring and the skin was draped with complete aseptic strict precautions.

A standard lumbar puncture was performed, usually in the L4-5 interspace, with a large-bore Tuohy needle (14-to 16-gauge). When CSF egress started, the direction of the needle was changed cephalad, the stylet was withdrawn, and the catheter was progressed into the subarachnoid space at least $20 \mathrm{~cm}$.

The catheter (17-or 18-gauge) was then slowly advanced with one hand, and the needle was removed at the same time. The drain was attached to an external drainage sterile container.

Sterile dressings were applied, a loop was made in the catheter to relieve tension, and the catheter was taped over onto the patient's flank. The lumbar drain was set to patients' shoulder level with complete bed rest afterwards.

Close monitoring especially to avoid overdrainage as common complication. The Lumbar Drain (LD) was set at shoulder level to drain 10 to $15 \mathrm{cc}$ per hour and approximately 300 to $400 \mathrm{cc}$ in the first two days.

Drainage was continued for 2 days postoperatively, after that the LD was closed for the next 24 hours and, if there no CSF leak, the LD was withdrawn on the fourth day.

Patients were discharged within 72 hours of stoppage of CSF leak and regular follow-up in outpatient clinic.

\section{Results}

Among (20) patients of cerebrospinal fluid leak collected in the study the mean age was (35.6 \pm 15.2$)$ years old ranged between 1 to 60 years, as regards gender; $12(60 \%)$ were males and $8(40 \%)$ were females. (Table 1$)$.

Table (1): Demographic characters of cases.

\begin{tabular}{cll}
\hline Variables & \multicolumn{2}{l}{ Number $(\mathrm{n}=20)$} \\
\hline Age groups: & 5 & $25 \%$ \\
<20 years & 11 & $55 \%$ \\
20-40 years & 4 & $20 \%$ \\
>40 years & & \\
Gender: & 12 & $60 \%$ \\
Male & 8 & $40 \%$ \\
Female &
\end{tabular}

As regards clinical presentation of patients 8 (40\%) of patients were presented by head trauma, and same percentage had skull base surgery, 4 $(20 \%)$ were presented by spine surgery. As regards etiology of leakage in $8(40 \%)$ of patients were post traumatic, and same percentage were suspected with skull base surgery, finally $4(20 \%)$ were suspected with intraduralspine surgery. For site of leakage higher percentage of $(70 \%)$ had rhinorrhea, $20 \%$ had leakage from operation incision, and $10 \%$ had CSF otorrhea. (Table 2).

Table (2): Clinical characters of cases.

\begin{tabular}{lcc}
\hline CSF leakage & \multicolumn{2}{c}{ Clinical characters } \\
\hline (n=20) & No. & $\%$ \\
\hline Type of patient: & & \\
Head trauma & 8 & $40 \%$ \\
Skull base surgery & 8 & $40 \%$ \\
Spine surgery & 4 & $20 \%$ \\
Etiology of leakage: & & \\
$\quad$ Post traumatic & 8 & $40 \%$ \\
Post-operative & 4 & $20 \%$ \\
Suspected post-operative & 8 & $40 \%$ \\
Site of leakage: & & \\
Rhinorrhea & 14 & $70 \%$ \\
Operation incision & 4 & $20 \%$ \\
Otorrhea & 2 & $10 \%$ \\
\hline
\end{tabular}

Majority of patients (80\%) were candidates for post-operative continuous lumber drain insertion, versus $20 \%$ who underwent pre-operative insertion. 
As surgical management for leakage most of patients underwent primary dural repair either with (80\%) graft of (fat, muscle, fascia, or pericranium) or $(20 \%)$ primary stitches. Four patients underwent endoscopic repair (Table 3).

Table (3): Ways of leakage management among cases.

\begin{tabular}{lll}
\hline \multirow{2}{*}{$\begin{array}{c}\text { Variables } \\
(\mathrm{n}=20)\end{array}$} & \multicolumn{2}{c}{ Frequency } \\
\cline { 2 - 3 } & No. & $\%$ \\
\hline Lumber drain insertion: & & \\
$\quad$ Continuous post-operative & 16 & $80 \%$ \\
$\quad$ Continuous pre-operative & 4 & $20 \%$ \\
Surgical management: & & \\
$\quad$ Dural repair & 16 & $80 \%$ \\
$\quad$ Endoscopic repair & 4 & $20 \%$ \\
Type of repair: & & \\
$\quad$ Graft \\
primary duralstitches
\end{tabular}

All patients complained of headache after operation, but $30 \%$ complained of headache and nausea, also $10 \%$ complained of headache, nausea and vomiting. Superficial wound infection was found in one patient that was managed conservatively by antibiotics and repeated dressings. No other complications were detected with regular follow-up for one month (Table 4).

Table (4): Complication and final outcomes among cases.

\begin{tabular}{lcc}
\hline \multirow{2}{*}{ Complications } & \multicolumn{2}{c}{ Frequency $(\mathrm{n}=20)$} \\
\cline { 2 - 3 } & No. & $\%$ \\
\hline Headache & 20 & $100 \%$ \\
Nausea & 6 & $30 \%$ \\
Vomiting & 2 & $10 \%$ \\
Superficial wound infection & 1 & $5 \%$ \\
\hline
\end{tabular}

Fifteen patients experienced excellent results with cessation of CSF leakage within 4 days of CLD, 4 patients experienced good results with cessation of CSF leakage within 5 days of CLD and only one patient experienced fair result which required re-exploration then CSF leakage stopped within 5 days of CLD Fig. (1).

\section{Statistical analysis:}

Data were gathered and coded to make manipulation of data easier and were double entered into Microsoft Access and data analysis was performed using Statistical Package of Social Science (SPSS) software version 18 in Windows 7. Qualitative data were analysed in a simple descriptive way in the form of numbers and percentages, and quantitative parametric data were analysed by arithmetic means as central tendency measurement, standard deviations as measure of dispersion.
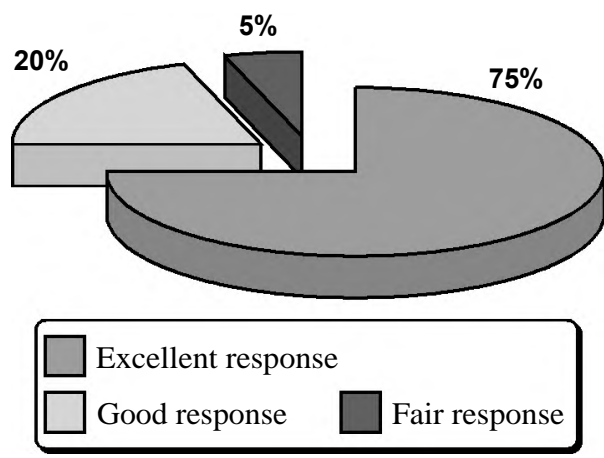

Fig. (1): Post-operative cessation of CSF leakage among cases.

\section{Discussion}

CSF leak is a very serious and dangerous problem which requires a careful management with close observation and follow-up. It predisposes to life-threatening conditions like bacterial contamination that can lead to threatening infections, especially meningitis. Therefore, they require thorough and timely evaluation and treatment $[4,5]$

CLD can be used for different objectives by neurosurgeons, such as in subarachnoid hemorrhage to prevent vasospasm, treating post-traumatic and post-operative CSF leak, and even reducing high intracranial pressure. Perioperative CSF diversion reduces the risk of post-operative CSF leaks. Lumbar drainage can be used as a prophylactic measure and/or as a definitive treatment option for CSF leak following surgery. LD can prevent and/or treat CSF leaks following skull base surgeries and may abandon re-exploration surgery [6,7]

The debate is about when to place the lumbar drain as some surgeons place them before surgery and others place them post-operatively only in the setting of a post-operative CSF leak. Furthermore, with the lumbar drains that were placed prior to surgery, there is usually no mention of whether the lumbar drain was actually placed in the preoperative period or placed after sedation and intubation of the patients.

Placing a lumbar drain in an intubated patient has a theoretical risk of injuring a nerve root or causing a spinal hematoma which would otherwise not be detected early. On the other hand, placement of lumbar drains are painful to patients so many surgeons may prefer to place them after patient sedation.

Our study included 20 patients with CSF leak either traumatic or post-operative. 12 patients $(60 \%)$ were males and 8 patients $(40 \%)$ were females. Ages of the patients ranged from 1 year to 60 years with mean age in the third decade. 
Paul et al., mentioned that in their study there were 20 males (49\%) and 21 females (51\%). 13 patients (14\%) were presenting with post-traumatic CSF leakage, 41 patients (44\%) were suspected to have post-operative CSF leakage. 23 patients $(24.7 \%)$ were operated upon by endoscopic approaches. 8 patients $(18.6 \%)$ experienced nausea and vomiting, two patients $(4.9 \%)$ complained of persistent CSF leak and required re-exploration. They also mentioned that CSF drainage is only started after observing those patients for 12-hour period in the neurosurgical ICU with the patient lying flat and the lumbar drain set at shoulder level. CT scan was done to exclude cerebral edema, hydrocephalus, tension pneumocephalus and ensuring patent basal cisterns. 10 to $15 \mathrm{cc}$ were drained per hour from 8:00 AM to 5:00 PM with meticulous neurological observation for three days. Drained was set to drain approximately $300 \mathrm{cc}$ of CSF over two days. On the fourth day the drain was closed and then removed after 24 hours of cessation of a CSF leak. Overall, they found that pre-operative lumbar drain placement is an effective and well tolerated means of preventing postoperative CSF leaks [8].

Pinar et al., mentioned in their study that the age of patients ranged from 8 months to 46 years with mean age in the third decade of life that was similar to our study. They declared that CLD is an essential tool to improve absorption capacity after endoscopic third vintriculostomy [9].

In 2007, Bien et al., performed a retrospective analysis of 150 posterior fossa craniotomy surgeries and noticed that placement of pre-operative LD decreased the incidence of post-operative CSF leak [10].

Most studies did not answer the question when to insert the LD; however, Ransom et al., placed the lumbar drain prospectively during surgery and Mehta et al., who reported the placement of the lumbar drain before surgery after patient intubation. No neurological deficits were recorded related to the lumbar drain insertion [11,12].

On the other hand, other authors use only place lumbar drains for a definite post-operative CSF leak is seen. However, Laws et al., may place a lumbar drain pre-operatively to facilitate delivering the suprasellar extension of macroadenomas. As of yet, there is no consensus on the timing of when a lumbar drain should be placed [13] .

Shapiro et al., and Esposito et al., [14,15] mentioned that in regards to the efficacy of lumbar drain CSF diversion in treating post-operative CSF leaks, it is generally considered an effective treatment for cerebrospinal fluid leak with success rate above $90 \%$.

In conclusion, the effectiveness of lumbar drain in this work was near excellent in cessation of CSF leak as the result was $95 \%$ which reached $100 \%$ after re-exploration and repair.

\section{Conclusion:}

Lumbar drains are used for perioperative CSF diversion as a prophylactic measure and a line of treatment for CSF leak. The risk of complication with the use of lumbar drains could be considered low. Although lumbar drain insertion increases the length of hospitalization, it could be considered safe in general with adequate precautions and close monitoring.

In conclusion, it is commonly suggested that perioperative continuous lumbar drainage is generally well-tolerated by patients and demonstrates that the effect of continuous lumbar drainage as a CSF diversion method decreases the overall risk of CSF leaks.

\section{References}

1- BRANDT M.T., JENKINS W.S., FATTAHI T.T. and HAUG R.H.: Cerebrospinal fluid: Implications in oral and maxillofacial surgery. J. Oral Maxillofac. Surg., 60 (9): 1049-56. doi: 10.1053/joms.2002.34419. [PubMed] [CrossRef], 2002.

2- CLEMENZA J.W., KALTMAN S.I. and DIAMOND D.L.: Craniofacial trauma and cerebrospinal fluid leakage: A retrospective clinical study. J. Oral Maxillofac. Surg., 53 (9): 1004-7. doi: 10.1016/0278-2391(95)90114-0. [PubMed] [CrossRef], 1995.

3- LOEW F., PERTUISET B., CHAUMIER E. and JAKSCHE H.: Traumatic, spontaneous and post-operative CSF rhinorrhea. Advances and technical standards in neurosurgery. Berlin: Springer, pp. 169-207. [PubMed], 1984.

4- FRIEDMAN J.A., EBERSOLD M.J. and QUASTLM: Post-traumatic cerebrospinal fluid leakage. World J. of Surg., 25 (8): 1062, 2001.

5- BELL R.B., DIERKS E.J., HOMER L. and POTTER B.E.: Management of cerebrospinal fluid leak associated with craniomaxillofacial trauma. J. Oral Maxillofacial Surg., 62 (6): 676-84, 2004.

6- DALGIC A., OKAY H.O., GEZICI A.R., DAGLIOGLU E., AKDAG R. and ERGUNGOR M.F.: An effective and less invasive treatment of post-traumatic cerebrospinal fluid fistula: Closed lumbar drainage system. Minim. Invasive Neurosurg., 51 (3): 154-7, 2008.

7- JUNG H., SHAH A. and AJLAN A.: Perioperative Cerebrospinal Fluid Diversion Utilizing Lumbar Drains in Transsphenoidal Surgery. J. Neurol. Disord., 2: 2-150, 2014. 
8- PAUL D., DREW A., SPENCER C. and PRABHU: The Efficacy and Safety of Preoperative Lumbar Drain Placement in Anterior Skull Base Surgery. J. Neurol. Surg. Rep., 74: 1, 2013.

9- PINAR O., JONATHAN R., LIANA B.A. and SHLOMI C.: Continuous spinal drain following endoscopic third ventriculostomy: A proposal to change the definition of failure. Childs Nerv. Syst., 27: 1973-8, 2011.

10- BIEN A.G., BOWDINO B., MOORE G. and LEIBROCK L.: Utilization of pre-operative cerebrospinal fluid drain in skull base surgery. Skull Base, 17: 133-9, 2007.

11- RANSOM E.R., PALMER J.N., KENNEDY D.W. and CHIU A.G.: Assessing risk/benefit of lumbar drain use for endoscopic skull-base surgery. Int. Forum. Allergy Rhinol., 1: 173-7, 2011.
12- MEHTA G.U. and OLDFIELD E.H.: Prevention of intraoperative cerebrospinal fluid leaks by lumbar cerebrospinal fluid drainage during surgery for pituitary macroadenomas. J. Neurosurg., 116: 1299-303, 2012.

13- LAWS E.R. Jr., De LOS REYES K. and RINCONTORROELLAJ: Lumbar drains intranssphenoidal surgery. J. Neurosurg., 118: 480-1, 2013.

14- SHAPIRO S.A. and SCULLY T.: Closed continuous drainage of cerebrospinal fluid via a lumbar subarachnoid catheter for treatment or prevention of cranial/spinal cerebrospinal fluid fistula. Neurosurgery, 30: 241-5, 1992.

15- ESPOSITO F., DUSICK J.R., FATEMI N. and KELLY D.F.: Graded repair of cranial base defects and cerebrospinal fluid leaks in transsphenoidal surgery. Neurosurgery, 60: 295-304, 2007.

\section{دور الدرنقة القطنية المستمرة فى تسرب السائل النخاعى،

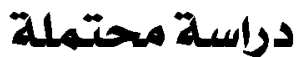

\footnotetext{
إن تسرب العائل النظاعى آحد آهم المثاكل التى قد تهدد حياة المرضى وقد تصل إلى الوفاة. يجب الفصص الطبى الدقيق وإجراء

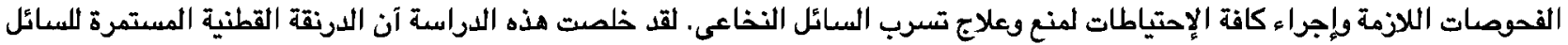

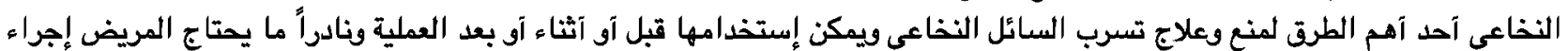
جراحة آخرى لعلاج تسرب السائل النخاعى.
} 